\title{
Electrically Isolated System for Measuring Signals in MHz-Band Human-Area Networks
}

\author{
Tomonori Nakamura, Mami Nozawa, Taku Kobase*, Hitoshi Simasaki, and Yuichi Kado \\ Department of Electronics, Kyoto Institute of Technology, \\ Kyoto, Japan \\ $+81-75-724-7451$ \\ kado@kit.ac.jp
}

\begin{abstract}
This paper presents a novel electrically isolated measuring system for evaluating signal attenuation on the human body and designing a stable MHz-band human-area network system that uses the surface of the human body as a data transmission path. We developed an electrically isolated probe consisting of an electrical-to-optical (E/O) converter and an optical-to-electrical $(\mathrm{O} / \mathrm{E})$ converter because there was a requirement for a tool to measure signal loss that did not affect our communication system. We modeled the communication path consisting of a transmitter, a receiver, and the human body. And we measured signal intensity to ensure an effect of the probe. As a result, the measurement system using an $\mathrm{E} / \mathrm{O}$ and $\mathrm{O} / \mathrm{E}$ conversion probe is effective to achieve a stable communication system.
\end{abstract}

\section{Categories and Subject Descriptors}

B.4.1 [INPUT/OUTPUT AND DATA COMMUNICATIONS]: Data Communications Devices - Receivers, Transmitters. B.4.5 [INPUT/OUTPUT AND DATA COMMUNICATIONS]: Reliability, Testing, and Fault-Tolerance-Diagnostics, Hardware reliability.

B.4.4 [INPUT/OUTPUT AND DATA COMMUNICATIONS]: Performance Analysis and Design Aids -Worst-case analysis.

\section{General Terms}

Measurement, Design, Reliability, Experimentation.

\section{Keywords}

Near-field coupling communication, Electrically isolated measurement, Path/Signal loss, Electrical-to-optical and opticalto-electrical conversion

\section{INTRODUCTION}

As concerns over safety, security, and health are growing in our aging society, wireless body area networks around the human body represent a promising technology for new applications in areas like secure systems, ITS, medical information systems, and payment systems. Body-channel communication (BCC) technologies have recently been actively reported [1]-[4]. However, these communication technologies are only composed of transceivers on the human body (wearable TRXs). We propose a human-area networking technology that consists of both wearable TRXs and TRXs embedded in environments or in equipment (embedded TRXs) to broaden the areas in which BCC can be applied [5]-[7]. The technology uses the human body as a communication path based on a near-field coupling mechanism using MHz-band.

Figure 1 shows typical applications of near-field coupling communication (NFCC). A user with a wearable TRX can communicate, recognize, and operate a smartphone with a TRX module embedded in it by holding it, and it locks when he/she loses his/her grip on it. When a user with a wearable TRX passes a ticket wicket, he/she can connect to an IP network and complete the payment process simply by using the electrodes of the embedded TRX installed on the ground of the ticket wicket, so he/she can easily pass through even when both his/her hands are occupied.

It is important to assess loss in the path from the transmitter to the receiver and model the communication path to achieve stable communications. We have already reported that a received power is overestimated when we directly measure the received signal of wearable TRXs with a conventional electric probe [8]. So, we present the measurements of signal attenuation using an electricalto-optical $(\mathrm{E} / \mathrm{O})$ and optical-to-electrical $(\mathrm{O} / \mathrm{E})$ conversion probe and demonstrate that they do not affect the communication system under test in this paper.

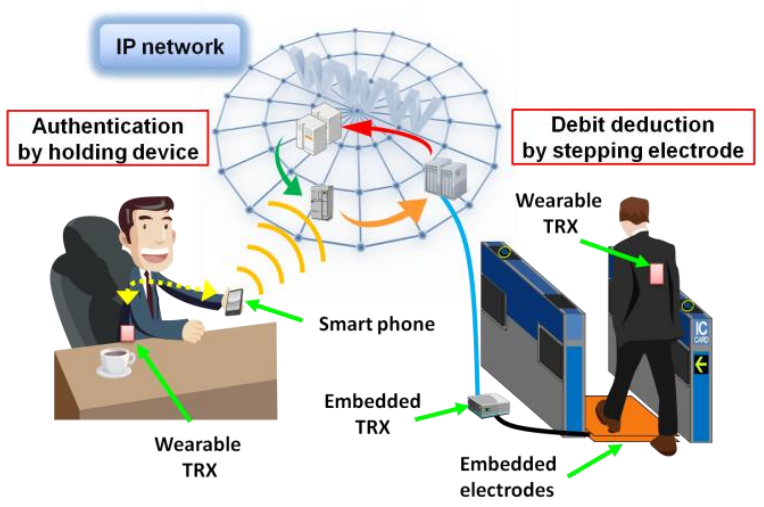

Figure 1. Typical applications of near-field coupling communications. 


\section{COMMUNICATION PATH MODEL}

The communication circuit model for the NFCC system is shown in Figure 2. The embedded TRX induces a modulated quasielectrostatic field signal on the human body, and communication occurs when the wearable TRX detects the signal from the embedded TRX. The signal loop consists of two types of paths. The first is a forward path and the second is a return path. The forward path is a route from the upper electrode of the embedded TRX to the electrode of the wearable TRX on the body side (signal electrode) through the human body. The return path is also a route from the electrode of the wearable TRX on the side opposite the body (ground electrode) to the lower electrode through earth grounding. We regard the human body as a conductor in the $6.75 \mathrm{MHz}$ frequency range used for communication. However, there are variable coupling capacitances occurred from clothes or shoes. Path loss depends on these capacitances. Therefore it is difficult to define path loss clearly by using a distributed constant or by analyzing the electromagnetic field. We need a tool to precisely measure the signal intensity on the communication path and to design stable communication network.

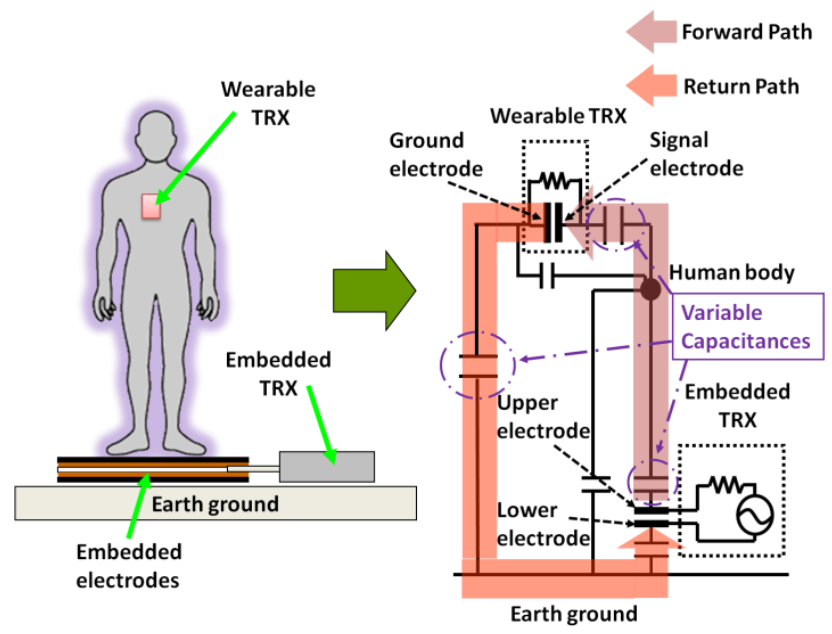

Figure 2. Communication circuit model.

\section{MEASUREMENTS OF SIGNAL INTENSITY}

\subsection{E/O and O/E Probe}

We designed and built a probe to accurately measure the received signal of a wearable TRX consisting of an E/O converter and an $\mathrm{O} / \mathrm{E}$ converter. Figure 3 has a block diagram of the probe. First, this probe converted the electrical signal to an optical signal and transmitted it to a measuring instrument in a transmission optical fiber. Next, it converted the optical signal to an electrical signal again. There is a photograph of the circuit E/O in Figure 4 (a). It receives the signal instead of the wearable TRX. It has two acrylic plates with electrodes, the driver for the laser diode (LD) module, and an amplifier circuit in it. The electrodes are parallel plates, and have same size as those of the wearable TRX. There is also a photograph of the circuit O/E in Figure 4 (b). An amplifier on the circuit amplifies the received signal with a photodiode. The signals between the two devices propagate in the optical fiber. In addition, the circuit $\mathrm{E} / \mathrm{O}$ is driven by a battery, and the circuit $\mathrm{O} / \mathrm{E}$ is driven by a DC source. The circuit gain of the $\mathrm{E} / \mathrm{O}$ and $\mathrm{O} / \mathrm{E}$ probe is $26.0 \mathrm{~dB}$ at $6.75 \mathrm{MHz}$.

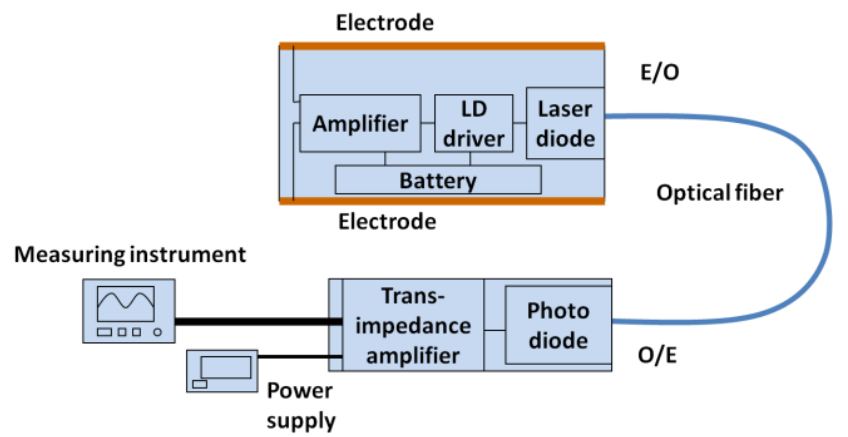

Figure 3. Block diagram of E/O and $\mathrm{O} / \mathrm{E}$ probe.

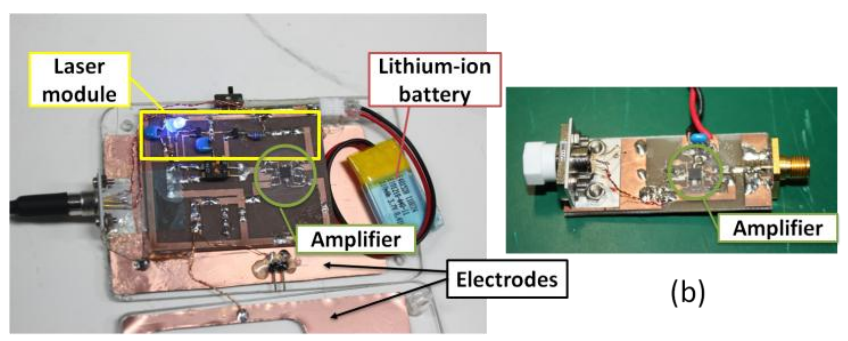

(a)

Figure 4. Photographs of E/O and O/E probe.

\subsection{Effect of Conventional Measurement}

\section{System}

The equivalent circuit on which we directly measured an electrical signal with the measuring instrument is shown in Figure 5. It adds coupling capacitance between the wearable TRX and earth grounding when the ground electrode of the TRX is connected to the housing of the measuring instrument. The height of the measuring instrument varies capacitance. Signals were strengthened when capacitance enhances the return path. We incorrectly measured the signal intensity due to the above. We used the following measuring system to actually confirm this effect.

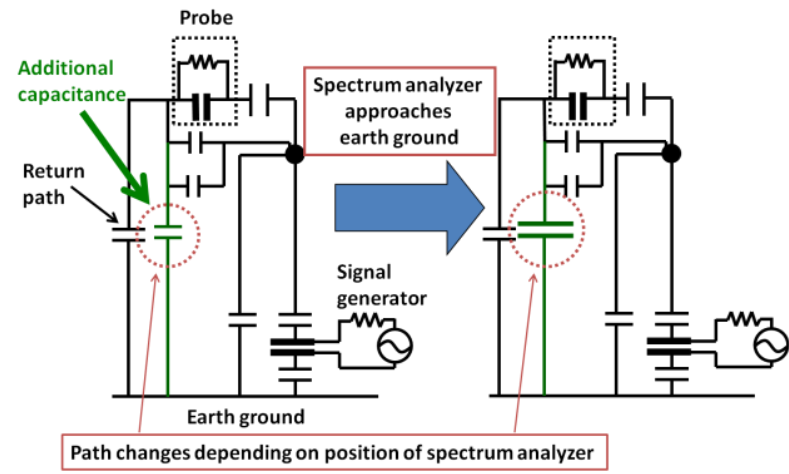

Figure 5. Equivalent circuit of conventional measurement system.

We measured signal voltages that the probe received when the ground electrode was directly connected to the measuring instrument and when it was not connected. The experimental system is outlined in Figure 6. We chose a spectrum analyzer as the measuring instrument, which was a battery-powered instrument that was independent of the earth grounding. We used two copper plates that were $350 \mathrm{~mm}$ square as electrodes for the embedded TRX. The distance between the plates was $10 \mathrm{~mm}$. We entered a sine wave of $6.75 \mathrm{MHz}$ by using a signal generator. We 
placed a phantom with the same electrical properties as the human body whose conductivity was $0.59 \mathrm{~S} / \mathrm{m}$ on a rubber plate against the upper electrode. The phantom was a rectangular solid filled with a gel material that absorbed water. It was $190 \mathrm{~mm}$ wide and long, and $950 \mathrm{~mm}$ high. The reason we used the phantom was to ensure the experiments could be reproduced. The ground electrode of the $\mathrm{E} / \mathrm{O}$ probe was connected to the spectrum analyzer with a lead line that was $1 \mathrm{~m}$ long and it was placed against the top of the phantom. We have plotted the ratio between applied and received voltages, and changes due to the height of the spectrum analyzer in Figure 7.

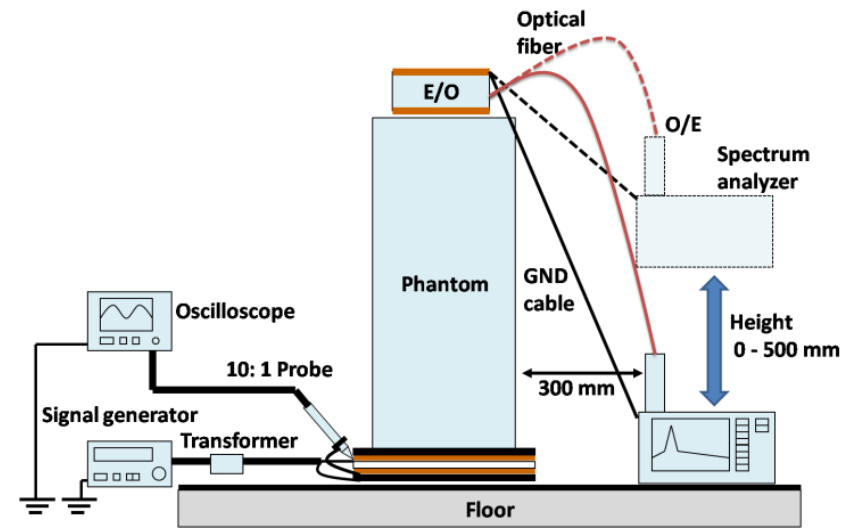

Figure 6. Experimental system to find effect on communication path.

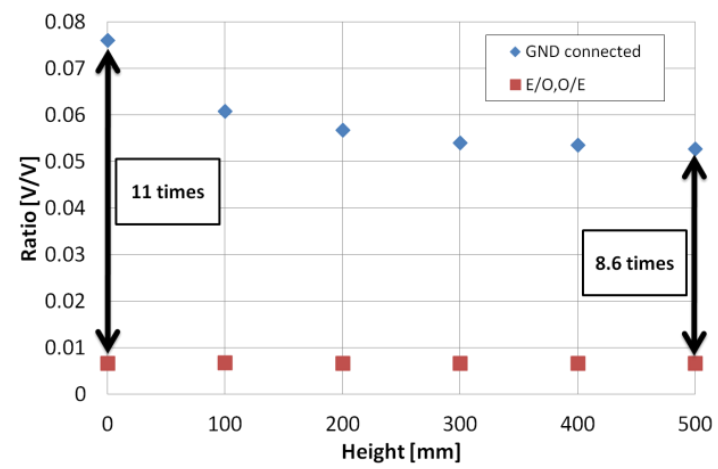

Figure 7. Ratio of applied voltages to received voltages as function of spectrum analyzer height.

There was about an 8.6 times difference when the spectrum analyzer was $500 \mathrm{~mm}$ high. The closer the spectrum analyzer was to the earth grounding, the larger the received signals were. In contrast, the measured values using the $\mathrm{E} / \mathrm{O}$ and $\mathrm{O} / \mathrm{E}$ probe were constant. These results revealed that the probe solved the problem that measuring signals with a conventional electric probe overestimated the signal intensity. As a result, we correctly measured received signals and built a reproducible measurement system with an $\mathrm{E} / \mathrm{O}$ and $\mathrm{O} / \mathrm{E}$ probe.

\section{EXPERIMENTS AND RESULTS}

We measured the signal attenuation caused between the transmitter and receiver, which mimicked two patterns in practical use. The first was assessed in an experiment on the received signal of wearable TRXs in the vicinity of the human body. This experiment replicated a user wearing TRXs in his/her chest pocket or pants pockets, or wearing them in a holder from the neck down. Figure 8 is a schematic of the experimental system. The signal was transmitted to the embedded electrodes from a signal generator through a transformer by a connection with coaxial cables. We measured the voltage of the embedded electrodes by connecting an oscilloscope in parallel with them. The oscilloscope had an output impedance of $1 \mathrm{M} \Omega$. The transformer was used to recreate the structure of the practical embedded TRX. The optical fiber extending from the $\mathrm{E} / \mathrm{O}$ probe was connected to the $\mathrm{O} / \mathrm{E}$ converter with $5-\mathrm{V}$ supply voltage, and we measured the voltage with the oscilloscope that had an output impedance of $50 \Omega$ by using a coaxial cable with a SubMiniature version A (SMA) connecter.

We measured the received voltage of $\mathrm{E} / \mathrm{O}$ probe and the voltage between embedded electrodes, and calculated the path loss. There were polystyrene boards with a thickness of about $2 \mathrm{~cm}$ (attenuator) between E/O probe and the phantom. Polystyrene boards had same electrical characteristics as air. Figure 9 shows the path loss between the embedded electrode and the E/O probe. The path loss increases as the distance between the phantom and the E/O probe increases. We can use this relationship to design human area network with necessary noise margin corresponding to various use case.

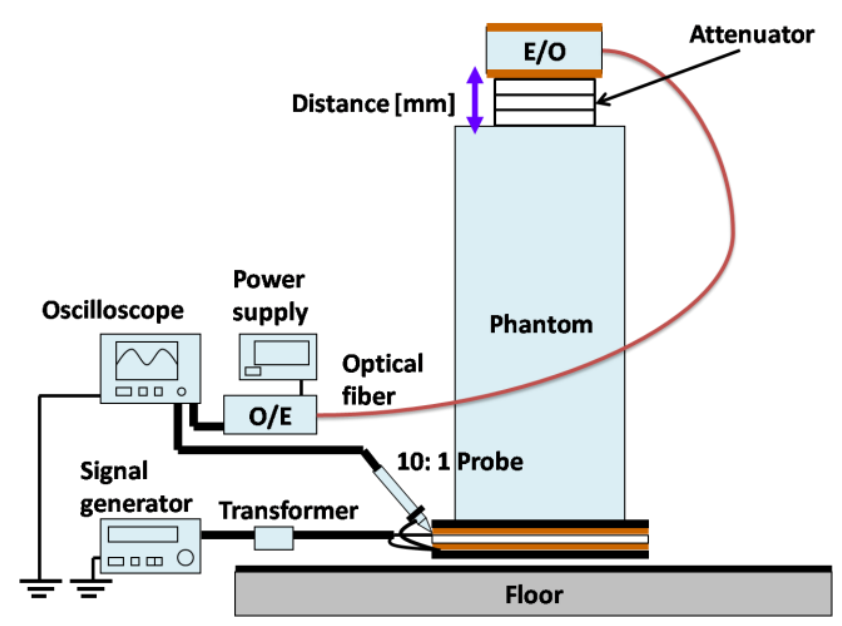

Figure 8. Measuring system for path loss.

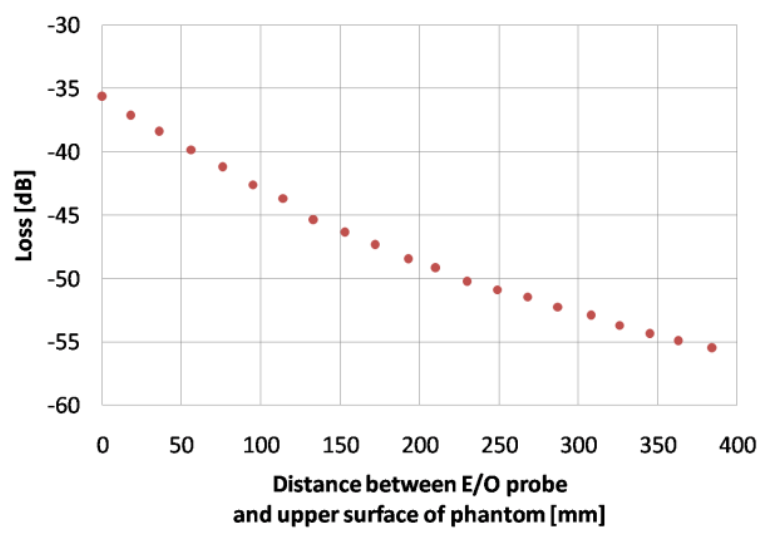

Figure 9. Path loss according to distance.

The second pattern is associated with interference between the user's TRX and other user's TRX. Figure 10 is a schematic of the experimental system where the phantom, embedded electrodes and probes are the same as those we used previously. We measured the received voltage of the surrounding phantom to assess the signal intensity around the phantom by using the E/O 
probe attached to a pole that was made of PVC pipe and was on wooden rails. In addition, the probe was always kept vertical to the ground. We also measured the difference in the potential of the embedded electrodes and drew a graph indicating vertical and horizontal attenuation depends on the distance from the phantom (Figure 11). As a result, we can confirm the signal intensity distribution around the phantom. This enables us to design stable communication network with minimal interference.

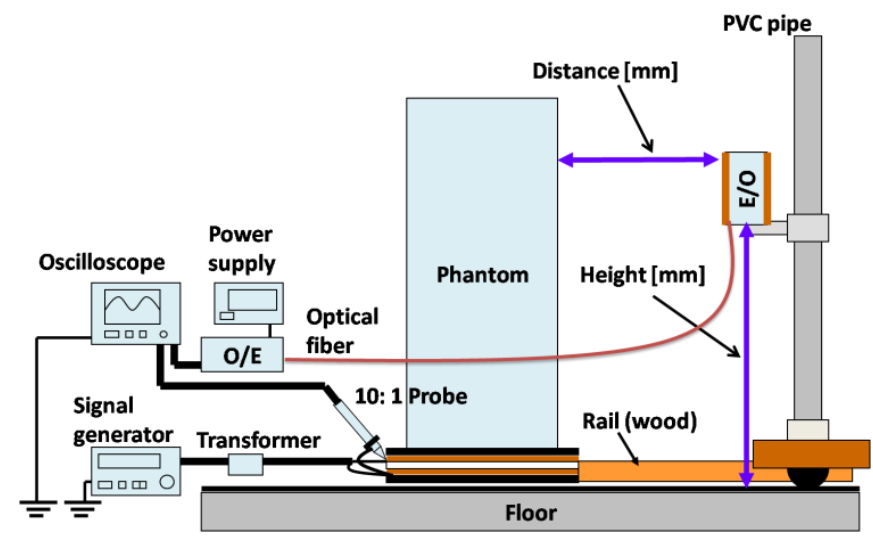

Figure 10. Measuring system for path loss around phantom.

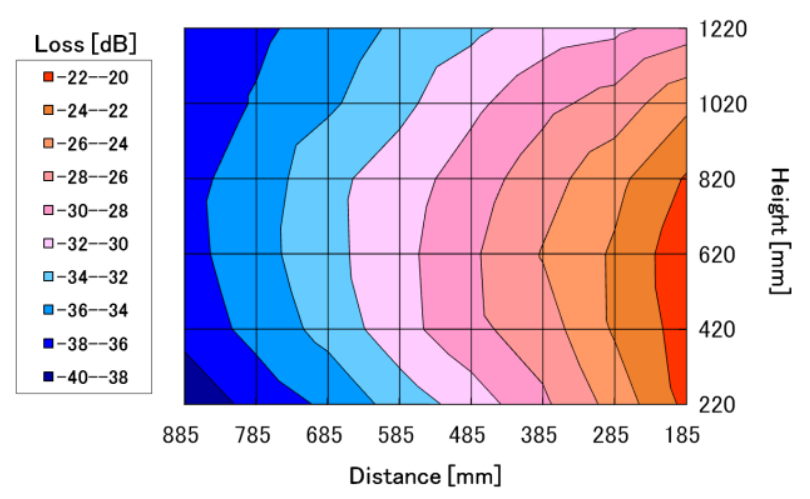

Figure 11. Path loss around phantom.

\section{CONCLUSION}

We proposed communication using NFCC between a transceiver on the human body and a transceiver embedded in environmental equipment. Our purpose was to precisely measure the signal intensity on the human body and assess signal loss. However, as there is a serious effect on the communication path by connecting the measuring instrument directly to the receiver, we proposed and implemented a method of measurement with the probe that was electrically isolated using $\mathrm{E} / \mathrm{O}$ and $\mathrm{O} / \mathrm{E}$ conversion. As a result, there was a voltage difference of more than $~ 8.6$ times in connecting the receiving electrodes to the housing of the measuring instrument.
We found that the new prove could solve the technical problem with overestimation of the received signal. We also measured signal attenuation in the vicinity of and around the human body using this probe. We achieved to assess loss of the communication path. Thus we can determine important factors of a transmission power or the sensitivity of the receiver by using the electrically isolated probe in order to produce a stable human centric communication system.

\section{ACKNOWLEDGMENTS}

Part of this work was supported by a Grant-in-Aid for Scientific Research (A) 23246073 from the Ministry of Education, Culture, Sports, Science and Technology of Japan.

\section{REFERENCES}

[1] N. Cho, J. Yoo, S. J. Song, J. Lee, S. Jeon, and H. J. Yoo,"The Human Body Characteristics as a Signal Transmission Medium for Intrabody Communication," IEEE Trans. Microwave Theory and Techniques, Vol. 55, pp. 1080-1086, May 2007.

[2] A. Fazzi, S. Ouzonov, and J. v. d. Homberg, “A 2.75mW Wideband Correlation-Based Transceiver for Body-Coupled Communication," IEEE ISSCC, pp. 204-205, Feb 2009.

[3] J. Bae, H. Cho, K. Song, H. Lee, and H. J. Yoo, "The Signal Transmission Mechanism on the Surface of Human Body for Body Channel Communication," IEEE Trans. Microwave Theory and Techniques, Vol. 60, pp. 582-593, March 2012.

[4] T. G. Zimmerman, "Personal Area Networks: Near-field intrabody communication," IBM Syst. J. , Vol. 35, no. 3--4, pp. 609--617, 1996.

[5] Y. Kado and M. Shinagawa, "AC Electric Field Communication for Human-Area Networking," IEICE Trans. Electron., Vol.E93-C, pp. 234-243, MAR 2011

[6] R. Nagai, T. Kobase, T. Kusunoki, H. Shimasaki, M. Shinagawa, and Y. Kado "Near-Field Coupling Communication Technology For Human-Area Networking," Proc. Conf. on Information and Communication Technologies and Applications (ICTA2011), International Institute of Informatics and Systems (IIIS), pp. 204-207, Orland, Nov. 2011.

[7] Y. Kado, T. Kobase, T. Yanagawa, T. Kusunoki, M. Takahashi, R. Nagai, O. Hiromitsu, A. Hataya, H. Shimasaki, and M. Shinagawa: "Human-Area Networking Technology Based on Near-Field Coupling Transceiver" 2012 IEEE Radio \& Wireless Sym. (RWS 2012), pp. 119 - 122, Santa Clara, California, USA, Jan. 2012.

[8] M. Takahashi, R. Shimoda, T. Kusunoki, T. Yanagawa, T. Kobase, R. Nagai, H. Shimasaki, and Y. Kado, "Characterization of MHz-Band Near-Field Coupling Communication Using Finite Element Electromagnetic Simulation", 6th European Conference on Antennas and Propagation (EUCAP), poster 01, Mar. 2012. 